EGU21-15258

https://doi.org/10.5194/egusphere-egu21-15258

EGU General Assembly 2021

(c) Author(s) 2021. This work is distributed under

the Creative Commons Attribution 4.0 License.

\title{
Impact of Climate Change on Coastal Meadows: a Mesocosm Approach
}

Thaisa Bergamo ${ }^{1,2}$, Raymond Ward ${ }^{1,2}$, Christopher Joyce ${ }^{2}$, and Kalev Sepp ${ }^{1}$

${ }^{1}$ Institute of Agricultural and Environmental Sciences, Estonian University of Life Sciences, Tartu, Estonia

(thaisa.fernandesbergamo@emu.ee)

${ }^{2}$ Centre for Aquatic Environments, University of Brighton, Brighton, United Kingdom

Coastal meadows supply a wide range of ecosystem services, including high carbon storage, high plant species richness and a wide variety of habitat types, which supports breeding and migratory bird populations. However, global change (climate change, pollution and environmental degradation) poses several threats to the stability and ecosystem services supplied by coastal meadows. Specifically within the Baltic Sea, recent estimates foresee various degrees of sea level rise along the Estonian coast and salinity is expected to decrease in the eastern Baltic and increase in the west. In order to assess the effects of climate change in coastal wetlands, an investigation of the influence of changes in water level and salinity on coastal wetland plant communities was undertaken. Future scenarios of Estonian coastal wetlands were evaluated using a three-year mesocosm experiment simulating altered environmental conditions. The response of three plant communities (Open Pioneer, Lower Shore and Upper Shore) were assessed in terms of changes in species composition through time. The experiment included 45 mesocosms, 15 per community with 5 treatments ( 3 replicates per treatment) with control, altered water level and salinity. Exploratory analysis, ANOVA and NMDS, were used to assess changes in the plant communities throughout the duration of the project. Preliminary results show that Open Pioneer is more sensitive to decreased salinity. A decrease in percentage cover of species adapted to high salinity concentration (e.g. Spergularia marina) was observed. On the other hand, Lower Shore didn't show any clear changes with the treatments. In order to obtain deeper insights, further analysis are needed to reveal complex community shifts under altered physical conditions. 